\title{
Management of functional gastrointestinal disorders
}

\author{
Authors: Asma Fikree ${ }^{A}$ and Peter Byrne ${ }^{B}$
}

Functional gastrointestinal (GI) disorders (eg irritable bowel syndrome and functional dyspepsia) are very common conditions which are associated with very poor quality of life and high healthcare utilisation. They are caused by disorders of GI functioning, namely altered gut sensitivity, motility, microbiota, immune functioning and central nervous system processing. They cause chronic symptoms throughout the gut (eg pain, dyspepsia and altered bowel habit), all of which are made worse by maladaptive patient behaviours, stress and psychological comorbidity. Management involves a biopsychosocial approach involving changes in lifestyle and diet, addressing coexisting psychological comorbidity and using medication to treat underlying pathophysiology. Pharmacological treatment with antispasmodics, neuromodulators, motility agents and antidepressants is effective. Psychotherapy in motivated individuals is equally effective. Success of treatment is increased by a good doctor-patient relationship and so this needs to be taken into account during the consultation.

KEYWORDS: gastrointestinal, irritable bowel syndrome, functional dyspepsia, functional disorders, pain

DOI: $10.7861 /$ clinmed.2020-0980

\section{Introduction}

Functional gastrointestinal disorders (FGID) are a group of disorders characterised by chronic gastrointestinal (GI) symptoms (eg abdominal pain, dysphagia, dyspepsia, diarrhoea, constipation and bloating) in the absence of demonstrable pathology on conventional testing. Historically, they were defined as conditions which had no organic basis, but this definition has evolved with increasing understanding of these conditions and we now know that they arise due to alterations in brain-gut communication. The current classification system (ROME IV) divides them into 33 adult disorders and 20 paediatric disorders, the most common subtypes

Authors: ${ }^{\text {A }}$ consultant gastroenterologist and honorary senior lecturer, Centre for Neuroscience, Surgery and Trauma, London, UK; ${ }^{\mathrm{B}}$ consultant liaison psychiatrist, honorary senior lecturer and associate registrar for public mental health, Royal London Hospital, London, UK, Centre for Neuroscience, Surgery and Trauma, London, UK and Royal College of Psychiatrists, London, UK being irritable bowel syndrome (IBS) which causes abdominal discomfort, altered bowel habit and bloating; and functional dyspepsia (FD) which causes epigastric pain or discomfort, often related to eating which can be associated with fullness and satiety.

\section{Epidemiology}

FGID are very common with a worldwide prevalence of $40 \%$, more common in women than men and this decreases with age. ${ }^{2}$ They account for $12 \%$ of the workload in primary care and $30 \%$ of gastroenterology outpatient consultations. 3,4 More than twothirds of patients with FGID will have seen a doctor in the last 12 months and $40 \%$ will use regular medication. ${ }^{5}$ FGID pose a huge economic burden and treating them cost the NHS at least $£ 72.3$ million in the year $2014 / 2015$, of which, two-thirds was on prescriptions, community care and hospital treatment. ${ }^{6}$

The presence of FGID is often associated with chronic pain (eg fibromyalgia) and other functional syndromes (eg chronic fatigue syndrome), and two-thirds will have psychopathology including anxiety and depression. ${ }^{7}$ It is therefore not surprising that these patients have very poor quality of life, worse than other chronic medical conditions (eg grade III congestive cardiac failure and rheumatoid arthritis). ${ }^{8}$

Due to the very low quality of life (QOL) of patients with FGID and the fact that they incur a lot of healthcare costs, it is important that they are recognised and managed promptly.

\section{Pathophysiology}

It is now clear that there is abnormal physiological functioning in patients with FGID, thought to be due to underlying alterations in GI motility (either too fast or too slow), visceral hypersensitivity, altered microbiota, increased intestinal permeability, low grade immune infiltration and altered central nervous system processing of sensory input. ${ }^{9}$ However, symptoms and healthcare seeking arise for complex reasons involving an interplay between early life events and coping styles, learned behaviour, alterations in GI physiology, and associated psychological morbidity as seen in the biopsychosocial model in Fig 1. ${ }^{9}$ Management is therefore not simply directed at the abnormal physiology or symptoms but has to address behaviours, cognitions and beliefs.

\section{Clinical approach}

\section{Assessment}

The optimal approach involves a holistic assessment starting with a detailed history, taking care to exclude the presence of red 


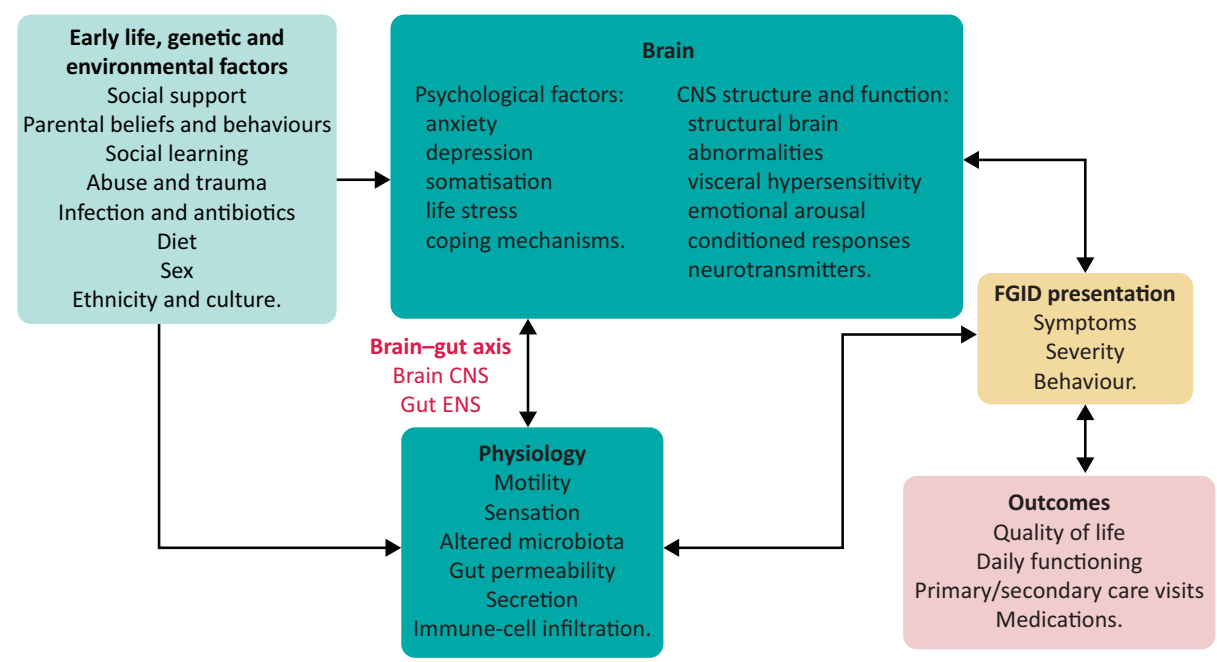

Fig 1. Biopsychosocial model of functional gastrointestinal disorders. CNS = central nervous system; ENS = enteric nervous system; FGID = functional gastrointestinal disorders. flags (weight loss, family history of cancer, nocturnal symptoms, anaemia or GI bleeding) and organic differentials. It is important to ask about diet, lifestyle and psychological status, as this will enable you to target these as part of the management plan. Integral to all this is the doctor-patient relationship and the quality of the consultation (Box 1). This involves being empathic, avoiding jargon, being honest and admitting when you do not have the answers, which takes a lot longer than organising yet another futile test. In our opinion, 10 minutes is not sufficient for this kind of consultation, however, spending time addressing all these factors on the first visit and breaking the diagnosis of a functional disorder will save time (and money) on future visits.

Examination should include an assessment for abdominal masses, and quality of pain as well as a rectal examination. The latter is essential to rule out rectal masses and haemorrhoids, and to assess for anal tone and function. The latter can be assessed at baseline and by asking the patient to squeeze as if they are preventing

\section{Box 1. Helpful questions during a consultation}

Can you tell me your story starting at the very beginning? This helps work out the chronology of symptoms and can be therapeutic for the patient.

What do you think is going on? This helps identify abnormal health beliefs.

What concerns you? This allows you to address this and can relieve some patient stress.

What do you want help with? If we could change one thing what would it be? This helps you focus the management plan around it.

What would you be doing in your life if these gastrointestinal symptoms were reduced? - This helps to identify an incentive for getting better, which could be a goal that the patient works towards.

Are there other things in your life that are stopping you from this? This helps identify psychosocial issues which are contributing to the symptoms and which may need to be addressed by their general practitioner. themselves from emptying their bowels. Anal hypotonia is associated with faecal incontinence and hypertonia can be associated with dyssynergic defecation, itself a cause of constipation.

\section{Investigations}

With the current ROME classification, it is possible to make a positive diagnosis of FGID based on the pattern of symptoms, and so exclusion of all organic disease is not necessary. However, serious differentials that seem feasible after taking a good history (Box 2) should be ruled out.

All patients should get a basic blood and stool panel including:

$>$ full blood count to look for anaemia

$>$ urea and electrolytes to look for dehydration and evidence of electrolyte derangements with diarrhoea

$>$ C-reactive protein or erythrocyte sedimentation rate to look for underlying inflammation, this should be normal in IBS

$>$ coeliac serology

$>$ thyroid function tests

$>$ faecal calprotectin, if diarrhoea is present, to rule out inflammatory causes of this

> Helicobacter pylori testing (stool antigen test or urea breath test) for patients with dyspeptic symptoms.

Endoscopy: If a patient has typical IBS symptoms with a normal faecal calprotectin and there are no red flags to suggest a colorectal cancer (see earlier) then a lower GI endoscopy is not needed. There is little yield in performing a gastroscopy for $\mathrm{H}$ pylori negative dyspepsia in the absence of alarm symptoms (such

\section{Box 2. Differentials for irritable bowel syndrome}

\section{Colorectal cancer}

Ovarian cancer

Bile salt malabsorption

Microscopic colitis

Coeliac disease

Crohn's disease 
continuous pain, vomiting, anaemia and weight loss in patients under the age of 60 ), so this should not routinely be organised. ${ }^{10}$

Abdominal ultrasound: Abdominal ultrasound can be useful in IBS to screen for abdominal causes of pain and, in particular, for ovarian cancer which can cause pain, visible abdominal bloating and altered bowel habit. In dyspepsia, it can be useful to look for gallstones if the history is suggestive (ie colicky pain with fatty meals).

SeHCAT scan. If available, SeHCAT scans should be used to assess for bile salt malabsorption which is present in up to a third of patients with IBS-D.11 Typical symptoms include watery diarrhoea, often yellow in colour, with or without nocturnal symptoms and faecal incontinence.

GI physiology. GI physiology is rarely indicated in IBS. One situation where it can be helpful is in patients who have severe constipation and are not responding to multiple laxatives. Lower GI physiology testing, particularly a colonic transit study and proctography can be useful at differentiating slow transit from a rectal evacuatory problem and can therefore help in fine tuning the management of constipation. In patients with functional dyspepsia, a gastric emptying study can be useful to look for severely delayed gastric emptying if there is persistent vomiting which is impacting on nutritional status, as this can help with decisions regarding feeding. For all physiological tests, it is important to be aware that medications, particularly opiates and anticholinergics, will alter GI motility and transit.

\section{Management}

\section{General and initial approach}

Once you have diagnosed a FGID, it is important to put a label on it, as patients often complain that they do not have a diagnosis or that 'nobody knows what is causing their symptoms', and then to explain in simple language what FGIDs are; information sheets or online resources can be very useful. In order to manage patient expectations, it is useful to reiterate the incurable nature of FGID and to explain that the aim of management is not to remove symptoms completely or return the patient back to 'normal', but to give them more control over their symptoms so that the GI symptoms do not dominate their life.

It is helpful when managing these patients to address the biopsycho-social factors, in reverse:

> social/lifestyle factors: diet, exercise, sleep, and ingestion of caffeine, alcohol and other medication

> psychological factors: presence of stress, anxiety, low mood and history of eating disorders

> biological factors: physiological abnormalities and medication side effects which are contributing to symptoms.

The heterogeneity of FGID makes it difficult to design an algorithm to fit all patients, however, using a biopsychosocial approach and identifying factors which may have triggered symptoms and which ones are maintaining them enables the clinician to focus on modifying these factors as part of a personalised management plan.

Integrated multidisciplinary clinical care (eg gastroenterologist, nurse, dietitian and psychologist) appears to be superior to gastroenterologist-only care in terms of improving symptoms, psychological state, quality of life and cost of treating functional gastrointestinal disorders, so this should be offered where possible. ${ }^{12}$

\section{Specific management}

Lifestyle advice

\section{Exercise}

Exercise can improve bowel function, improve transit time (in females) and, therefore, help with constipation. ${ }^{13}$ It can also reduce stress, improve mood and lead to better sleep, all of which impact on GI symptoms. In a randomised controlled trial (RCT), increased physical activity was associated with a greater reduction in IBS symptom severity scores. ${ }^{14}$ Patients should be advised to do 20-30 minutes of exercise 3-5 times per week, even if this is just walking.

\section{Sleep}

Sleep disturbances are associated with both upper and lower GI symptoms and worse QOL. ${ }^{15}$ Sleep disturbances can be initial (anxiety preventing onset of sleep), middle (typically broken sleep) and late (early morning awakening (can be a sign of depression)). Digital technology (eg 'Fitbits' and phone apps) can measure sleep patterns and quality, and then advise interventions to improve this (eg Sleepio app). Patients should be advised on sleep hygiene. If a drug is needed, benzodiazepines should be avoided but melatonin ( $3 \mathrm{mg}$ nocte) can be used. This has been shown to reduce abdominal pain and rectal hypersensitivity but not bloating or overall IBS symptom scores. ${ }^{16}$

\section{Phone apps}

Apps can be useful for tracking lifestyle factors and monitoring changes to this. There are hundreds of healthcare apps available so it can be difficult to know what to recommend. Apps which are listed on the NHS Apps Library or ORCHA (www.orcha.co.uk) have been reviewed and can be more confidently recommended. We tend to recommend Sleepio for sleep and Headspace for mindfulness. Bowelle helps track symptoms in IBS. Zemedy is an IBS self-management app that uses a cognitive behavioural therapy (CBT) approach. The Monash University Low FODMAP Diet $^{\text {TM }}$ App helps users identify foods with high fermentable oligo-, di-, and monosaccharides and polyols (FODMAP) content and is useful for patients who are on the low FODMAP diet (further details follow later).

\section{Stimulants and depressants}

Caffeine increases diarrhoea, alcohol worsens reflux symptoms and heavy use is associated with increased risk of functional dyspepsia. ${ }^{17}$ Both caffeine and alcohol can lead to disrupted sleep, so these should be minimised and not taken late at night in symptomatic individuals.

Cannabis misuse, with direct causative links to vomiting syndromes, has latterly been replaced by internet-bought cannabidiol and other plant derivatives. Even if the patient experiences none of the commonly associated GI side effects, such as vomiting and diarrhoea, there is an association with anxiety, fatigue, somnolence, risk of dependence and the potential for drug interactions, so this should not be recommended. ${ }^{18,19}$

\section{Diet and nutrition}

\section{Eating behaviours}

In FGID, symptoms are frequently associated with food intake, and so dietary assessment and optimisation should be part of the 
initial management strategy. Patients can be asked to keep a food diary to identify foods which trigger symptoms and to identify eating behaviours, however, the possibility of reporting bias should be noted.

IBS is associated with irregular dietary patterns and reduced diet quality and diversity (ie not having the recommended range and quantity of particular food groups), so education about how to eat as well as what to eat is important. ${ }^{20,21}$ National Institute for Health and Care Excellence (NICE) has a useful information sheet for an 'IBS diet' which focuses on eating small regular meals, avoiding insoluble fibre, fatty foods, gas producing foods and caffeine.

\section{Dietary restriction}

There is evidence for symptom improvement following reduction in lactose and in high-starch and sugary foods and drinks, so these can be reduced if appropriate. ${ }^{20}$

The identification that consumption of foods high in FODMAP can exacerbate symptoms of IBS because of fermentation and osmotic effects (bloating/pain and diarrhoea, respectively) has led to the use of a low FODMAP diet as a dietary intervention for IBS, particularly in IBS-D. ${ }^{22}$ However, this diet runs the risk of being overly restrictive and the long-term effects on nutrition and the colonic microbiome are unclear, so it is important that this is implemented by a trained dietitian. ${ }^{23}$

In two RCTs in Sweden and the USA, there was a similar reduction $(40-50 \%)$ in IBS symptom scores in both a 'low FODMAP diet' and an IBS/NICE diet. ${ }^{24,25}$ Thus, in the absence of dietetic support for the former, it is useful for a clinician to provide generic dietary advice in clinic based on NICE guidance.

The evidence for a gluten free diet is less clear cut. While minimising gluten intake is associated with a reduction in abdominal pain, it is unclear whether this is due to the fact that gluten is a high FODMAP food. ${ }^{26}$ Patients can be asked to reduce their gluten intake if this is felt to be helpful but there is no clear evidence for restricting this completely.

\section{Fibre}

Soluble fibre - psyllium or isphagula husk (eg fybogel) - is cheap and useful for symptoms of IBS, particularly in IBS-C. This should be started at low dose and increased slowly. There is no evidence for insoluble fibre or for bran, both of which can exacerbate pain and bloating. ${ }^{27}$

\section{Probiotics}

Patients with FGID have altered microbiota, and this will be increased if patients have been on long-term or recurrent antibiotics. Microbial alteration with probiotics can be trialled and there is growing evidence for this. A meta-analysis demonstrated that probiotics improve global IBS symptom scores as well as individual symptoms of abdominal pain, bloating and flatulence. ${ }^{28}$ Probiotics can improve stool consistency and frequency in both IBS-D and IBS-C but this is not as clear-cut as its effect on pain and bloating. ${ }^{29}$ Combination probiotics are more likely to be beneficial but there is no consistent data to suggest what that combination should be, nor what the dose should be. ${ }^{30}$ Although the long-term effect of probiotics on the microbiota is unknown, they are generally safe and so trialling them for 2-3 months can be considered early on in the treatment of IBS-D and possibly IBS-C.
There is no evidence to recommend faecal microbiota transfer, prebiotics (dietary supplements that result in specific changes in the composition and/or activity of the GI microbiota) or synbiotics (a mixture of probiotics and prebiotics that act synergistically to promote the growth and survival of beneficial organisms) at the current time.

\section{Psychopathology: identification and psychological treatment}

Although it may not be constructive to medicalise stress and distress by imposing diagnoses of anxiety and clinical depression, it is important to identify these as they are treatable and improving them will lead to an improvement in GI symptoms and QOL. Use of simple questionnaires (such as the hospital anxiety and depression scale) are a quick and easy way to identify this in an outpatient setting. Treatment is with pharmacological agents or with psychotherapy, both of which are effective treatments for FGID. ${ }^{31}$

\section{Anxiety}

Several symptoms of anxiety are similar to those in FGID (eg diarrhoea, vomiting, abdominal cramps and nausea) and patients with multiple functional syndromes often have an element of health anxiety. GI specific anxiety can be measured by the visceral sensitivity index (VSI) and this is the best predictor of IBS symptom severity.

\section{Depression}

It is important not to blame the GI disorder on low mood but explain how mood will contribute to GI symptoms and how it is treatable. Urgently refer patients with current suicidal ideation or plans to mental health professionals. For others, consider the role of psychotherapy versus pharmacotherapy for treating the low mood. As a general rule, unless there is suicidal ideation, we would consider psychotherapy (further details follow later) as a first line before medication.

\section{Eating disorders and disordered eating}

Although FGID are not typically associated with eating disorders, the presence of meal related symptoms can lead to disordered eating and, in severe cases, the development of food phobias. This is due to the conditioned pairing of an unpleasant GI symptom (eg abdominal pain) with specific foods which can then lead to avoidant restrictive food intake disorder (ARFID) which is an emerging category of eating disorders. ${ }^{32}$

\section{Psychotherapy}

A meta-analysis has confirmed that psychological therapies including CBT, gut-directed hypnotherapy, dynamic psychotherapy, and relaxation and mindfulness therapy are effective treatments for FGID (number needed to treat (NNT) 3-6). ${ }^{30}$ Which of those is chosen will be determined by availability of local services. CBT is probably the easiest type of therapy to access (via Improving Access to Psychological Therapies (IAPT)) and most effective with an NNT of 3, meaning at least one in three referrals to CBT will experience significant reduction in symptoms. ${ }^{31}$ Success 
of psychotherapy may be dependent on the expertise of the therapist; in some studies, psychotherapy delivered in specialised centres appears to have a better outcome. ${ }^{33}$ However, CBT and mindfulness is effective even when delivered via the internet which will improve access to psychological therapies. ${ }^{34,35}$ Psychotherapy can be time consuming, usually delivered over 12-14 weekly sessions. However, the effects appear to be long lasting and relatively free of side effects, so this form of therapy should be considered in willing patients. ${ }^{36-39}$

A meta-analysis has confirmed that antidepressants are also effective treatments for IBS, equally effective to psychotherapy, when patients are compliant with them. ${ }^{31,40}$ The use of antidepressants for treating symptoms of FGID are discussed later.

\section{Pharmacotherapy}

Biological management of FGID involves either treating the underlying pathophysiology (ie neuromodulators to treat visceral hypersensitivity) or treating the symptoms (eg antiemetics to treat nausea or laxatives to treat constipation). Various algorithms exist to help guide this for functional dyspepsia and for IBS (Fig 2). ${ }^{10,41}$ For the purposes of this review, a symptom-based approach is presented.

\section{Pain}

Opiates should be avoided as they are associated with dependence, tolerance and addiction, and can led to narcotic bowel syndrome which causes bloating, constipation, nausea and a paradoxical increase in pain with increasing doses of opiates. ${ }^{42}$
Instead, first-line management of pain involves antispasmodics for colicky pain. In more resistant cases, often characterised by chronic burning/neuropathic pain, neuromodulators are used as second-line treatment.

\section{First line: antispasmodics}

Antispasmodics are useful for colicky abdominal pain in IBS. There is good evidence for hyoscine (10-20 mg three times a day (tds); NNT 3) and dicycloverine (10-20 mg tds; NNT 4), though these can cause anticholinergic side effects of dry eyes and a dry mouth, and can worsen constipation. There is also evidence for peppermint oil (eg colpermin 2 capsules tds; NNT 4). It can cause heartburn, so may be best avoided for patients with coexisting GORD. ${ }^{30}$ The evidence for mebeverine (135 mg tds) is not as good, however, it is well tolerated and can be very effective in a group of patients which do not respond to other antispasmodics. ${ }^{30}$ Our practice is to trial a second antispasmodic if the first fails to control colicky abdominal pain.

\section{Second line: neuromodulators}

Antidepressants. The most commonly used antidepressants are tricyclic antidepressants (TCAs) and selective serotonin reuptake inhibitors (SSRIs). As a rule, antidepressants improve symptoms, particularly abdominal pain, as well as psychological distress in FGID, and the effect is increased in secondary compared with primary care, which probably reflects the greater psychological and pain comorbidity in the former. ${ }^{31}$ For every four patients treated with an antidepressant, one will get better.
Fig 2. Algorithms for pharmacological management of irritable bowel syndrome and functional dyspepsia. a) Irritable bowel syndrome. Dotted red boxes identify medications that are useful for more than one symptom in IBS-D, dotted blue boxes identify medications that are useful for combination symptoms in IBS-C. b) Functional dyspepsia. Dotted blue boxes identify medicines that target for both nutrient tolerance and pain. PPI = proton pump inhibitor; SNRI = serotonin noradrenergic reuptake inhibitor; SSRI = selective serotonin reuptake inhibitor; TCA = tricyclic antidepressant.

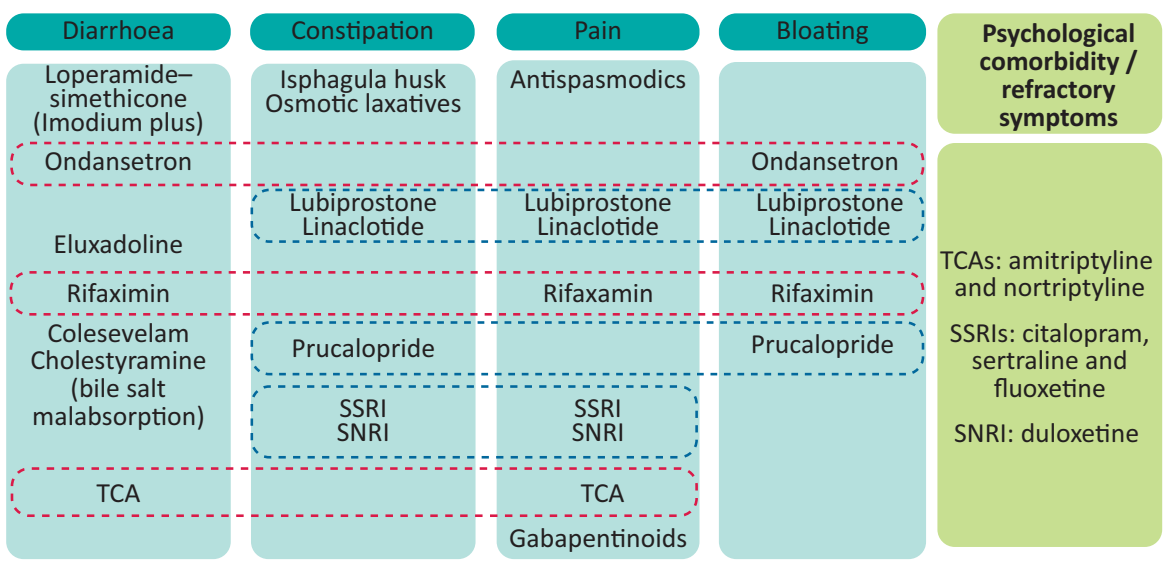

b

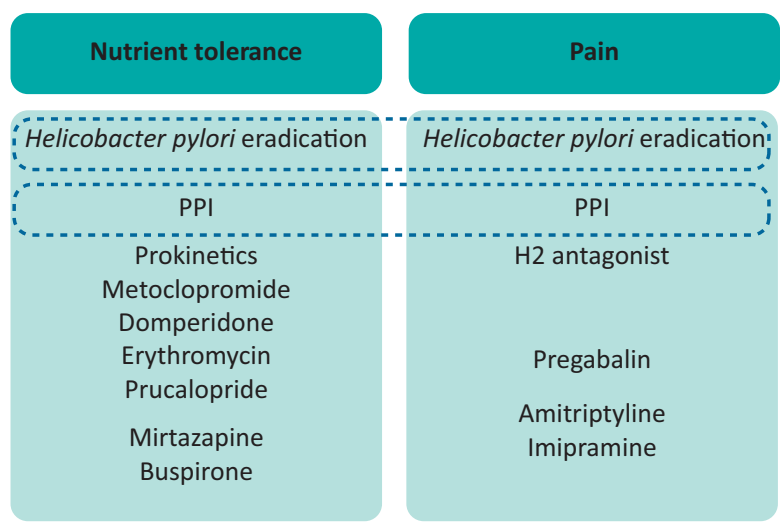

Psychological comorbidity / refractory symptoms

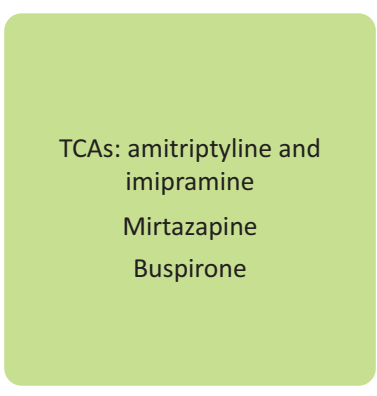


Most antidepressants will have GI side effects and can cause either constipation or diarrhoea (supplementary material S1), so choosing your antidepressant wisely can also help treat the altered bowel habit in IBS. TCAs (eg amitriptyline) are useful for patients with diarrhoea by slowing GI transit, whereas SSRIs (eg sertraline, citalopram, fluoxetine) are useful in those with constipation by accelerating transit. There are no RCTs of serotonin noradrenergic reuptake inhibitors (eg duloxetine) in FGID, however, open label studies for patients with IBS and comorbid anxiety and depression show that it is well tolerated at a total dose of $60 \mathrm{mg}$ per day and improves IBS symptoms as well as depression and anxiety. ${ }^{43,44}$

Treatment with antidepressants is for an average of 18 months and stops once patients are symptom free for at least 6 months. In order to improve compliance, it is important to clarify to the patients that antidepressants are used primarily to target the IBS symptoms, notably pain, rather than the mood. It is important to pre-warn patients about the side effects and, if necessary, start at a very low (subtherapeutic) dose and work up slowly, in order to reduce adverse effects and therefore improve compliance. We would caution against using more than one antidepressant at a time unless there is expertise in doing this.

Gabapentinoids. Pregabalin and gabapentin are commonly used in chronic pain conditions and have a role in treating visceral hypersensitivity in FGID. Pregabalin is associated with an improvement in IBS symptoms (bloating, diarrhoea and abdominal pain) compared with placebo, so is a good choice for IBS-D. ${ }^{45}$ This should be started at a low dose of $50 \mathrm{mg}$ twice a day (bd) and increased gradually in line with symptomatic response, to a maximum of $300 \mathrm{mg}$ bd, although it is not typically necessary to increase beyond $225 \mathrm{mg}$ bd $\left(3 \times 75 \mathrm{mg}\right.$ tablets bd). ${ }^{45}$ It causes weight gain, so may not be the best choice in obese individuals. Clinicians should be aware that it is addictive and is now considered a drug of abuse, so should be chosen with caution in certain patients. Gabapentin is an alternative but tends to have a worse side effect profile. Although there are no RCTs evaluating the efficacy of gabapentin on IBS symptoms nor the optimal dose for symptom improvement, one study demonstrated that, for patients using $300 \mathrm{mg}$ gabapentin daily, there was a reduction in rectal sensitivity to distension and an increase in the thresholds for abdominal pain, bloating and discomfort. ${ }^{46}$

\section{Diarrhoea}

\section{First line: loperamide}

Loperamide (Imodium) reduces stool frequency and improves stool consistency in IBS-D, however, it is not effective at reducing abdominal pain or bloating, so it is poorly tolerated in IBS patients. ${ }^{30}$ Loperamide-simethicone chewable product (Imodium plus) is much better tolerated and results in quicker relief from diarrhoea and greater relief from abdominal discomfort compared with loperamide alone or a placebo and, therefore, this should be preferentially recommended to patients who have IBS-D. ${ }^{47}$ Patients can be asked to take two tablets to start with and then one tablet after each unformed stool until the diarrhoea stops (up to a maximum of eight tablets per day).

\section{Second line: ondansetron}

Ondansetron, a 5HT3 antagonist, improves stool consistency, frequency, urgency and bloating but not abdominal pain compared with placebo or to mebeverine for patients with IBS-D, so it is especially useful for patients who are more troubled with the altered bowel habit than the pain. ${ }^{48}$ As it is an antiemetic, it can also improve nausea for patients who have overlapping dyspepsia. Alosetron was the precursor to ondansetron for IBS-D, however, it was associated with ischaemic colitis and therefore withdrawn. In a meta-analysis of patients with IBS-D and IBS-M, patients on $5 \mathrm{HT} 3$ antagonists did better than those on eluxadoline and rifaximin (see later), so it is worth considering the use of ondansetron early for patients with IBS-D. ${ }^{49}$

\section{Third line: rifaximin and eluxadoline}

Rifaximin is a non-absorbable antibiotic used in the treatment of GI disorders. A meta-analysis demonstrated that rifaximin is superior to placebo for reducing symptoms of diarrhoea and bloating in non-constipated patients with IBS, however, it had no effect on pain. ${ }^{30,50}$ Recent unpublished evidence suggests that with $550 \mathrm{mg}$ bd for 2 weeks, this effect may be sustained for at least 12 weeks after taking the antibiotic. However, rifaximin remains unlicensed for use in IBS in the UK and the long-term effects on the microbiota are unknown, so it is difficult to make evidence-based recommendations about this antibiotic at the current stage.

Eluxadoline is an opioid receptor antagonist which reduces stool frequency and improves global IBS symptoms, however, because of the association with pancreatitis, it is avoided for patients with a risk factor for acute pancreatitis (eg a prior history of pancreatitis, previous cholecystectomy, gallstones or alcohol). ${ }^{30}$ The RELIEF RCT demonstrated that, in non-responders to Imodium who have an intact gallbladder, eluxadoline can be helpful in reducing stool frequency and improving pain over a 12 -week period compared with placebo. A typical dose is $100 \mathrm{mg}$ bd but this can be reduced to $75 \mathrm{mg}$ bd in the case of side effects (eg nausea, abdominal pain, constipation and vomiting). ${ }^{51}$

\section{Constipation}

\section{First line: osmotic laxatives}

Polyethylene glycol (PEG)-based laxatives (such as Movicol and Laxido) as well as lactulose help to draw water into the bowel to soften the stool. Although they are associated with an increase in frequency of bowel movements, they do not alleviate pain in IBS. Lactulose can make bloating worse. In practice, it is useful to use osmotic laxatives to improve stool frequency for patients with constipation/IBS-C but this would need to be coupled with other agents to help with other symptoms such as pain.

\section{Second line: prucalopride}

Prucalopride is a highly selective $5 \mathrm{HT} 4$ agonist which acts as a prokinetic in the gut. An RCT demonstrated its efficacy in all patients with chronic constipation and in women with chronic constipation in whom laxatives have failed to provide adequate relief. ${ }^{52}$ According to NICE, it is licensed for women in whom treatment with two laxatives have failed. A typical dose is $2 \mathrm{mg}$ daily. A higher dose of $4 \mathrm{mg}$ daily will result in improvement in straining than in stool frequency but will also be associated with greater side effects (such as headache, nausea and diarrhoea). ${ }^{52,53}$ Patients who do not respond in the first 4 weeks are unlikely to do so with more treatment, so this can then be stopped. Prucalopride also acts as a gastric prokinetic, so it can be useful for patients with dyspepsia/ gastroparesis type symptoms and would be a good choice for patients who have IBS-C and functional dyspepsia overlap. 


\section{Third line: secretagogues}

If the above measures fail, then the third-line option involves the use of secretagogues (linaclotide and lubiprostone) which improve bowel frequency and overall IBS symptoms. Some patients experience unwelcome diarrhoea with this so it may be worth reducing the dose in that case.

Linaclotide results in increased chloride and bicarbonate secretion into the gut lumen which leads to increased fluid secretion and intestinal transit. It improves bowel frequency and reduces bloating compared with placebo; this effect is similar even if the dose $(290 \mu \mathrm{g})$ is reduced to $72 \mu \mathrm{g}$, which reduces diarrhoea (a common cause for discontinuation) and therefore may improve compliance. ${ }^{54,55}$

Lubiprostone at a dose of $8 \mu \mathrm{g}$ bd improves abdominal pain, bloating and stool frequency in IBS-C more than placebo but can be associated with nausea. ${ }^{56}$

\section{Functional dyspepsia}

This is the second most common FGID and can be divided into epigastric pain syndrome (EPS), characterised by epigastric pain and burning unrelated to meals, and postprandial distress syndrome (PDS) which causes early satiety, postprandial fullness, nausea and epigastric bloating. A proportion of patients with FD will also have mild to moderate delays in their gastric emptying (Fig 2b).

\section{First line: proton pump inhibitors and $\mathrm{H}$ pylori eradication therapy}

A meta-analysis showed that $H$ pylori eradication provides significant symptomatic benefits in the long term ( $>6$ months) rather than short term ( $<6$ months); but patients with functional dyspepsia are more likely to experience side effects including diarrhoea. It is therefore important to encourage them to remain compliant and complete the course and to ensure eradication following this if they have ongoing symptoms.

Patients who are proton pump inhibitor (PPI) responsive should be continued on the lowest dose needed to manage symptoms, and if this is not effective, it should be stopped. ${ }^{10}$

\section{Second line treatment: $\mathrm{H} 2$ blockers and prokinetics}

$\mathrm{H} 2$ blockers. In a meta-analysis of treatments for functional dyspepsia, $\mathrm{H} 2$ antagonists (such as ranitidine) are comparable to, if not more effective than, PPIs. This is not surprising in view of the increasingly important role played by mast cells and histamine in the development of functional dyspepsia. ${ }^{10}$ Translating this to clinical practice, it is worth trialling $\mathrm{H} 2$ blockers for patients with FD even when PPIs have failed, although, in the current climate, the supply issues with these medications may make this difficult in practice.

Prokinetics. Prokinetics can be used in PDS, particularly if there is delayed gastric emptying. They improve symptoms but not quality of life. ${ }^{57}$ Many prokinetics represented in RCTs are not available in the UK (eg itopride, acotiamide, cisapride and mosapride), however, these were no more effective than domperidone, which is available in the UK. Domperidone has been associated with increased QT intervals which has limited its use, however, for patients with a normal QT interval, domperidone can be safely trialled, although it is important to recheck electrocardiography once the patient is established on domperidone. There are no trials assessing other prokinetics (such as metoclopramide and erythromycin) in FD, however, theoretically these can be used in the short term.

\section{Third line: neuromodulators}

The evidence for antidepressants in functional dyspepsia is less clear but there seems to be a role for low dose TCAs such as amitriptyline (10-30 mg nocte) or imipramine ( $25 \mathrm{mg}$ daily for 2 weeks then $50 \mathrm{mg}$ daily) for patients with epigastric pain (ie epigastric pain syndrome). ${ }^{58,59}$ Patients need to be warned about anticholinergic side effects, which can reduce compliance. Mirtazapine is increasingly gaining popularity, especially for patients with postprandial symptoms (ie discomfort, fullness and nausea post-meals). It improves symptoms and nutrient tolerance even in the absence of coexisting depression and anxiety and causes weight gain, so would be ideal for patients with symptoms of postprandial fullness who are underweight. ${ }^{60}$ It also improves sleep and mood which will have a beneficial effect on global symptoms. Patients should start at $15 \mathrm{mg}$ nocte and increase monthly to a maximum of $45 \mathrm{mg}$. A meta-analysis has demonstrated that there is no role for SSRIs (eg sertraline, fluoxetine or citalopram) in FD. ${ }^{58}$ Buspirone is an anxiolytic which can improve gastric accommodation and can be useful for patients with postprandial fullness and early satiety but is associated with poorly tolerated side effects of dizziness and somnolence so not the first choice of neuromodulator. ${ }^{10}$

\section{Conclusion}

FGID are common but complex disorders which are associated with a lot of morbidity and associated psychopathology. As the aetiology of these disorders is still incompletely understood there remains no cure for them. Treatment involves a good therapeutic relationship and a holistic approach to treat the patient rather than disease, using a biopsychosocial model.

\section{Supplementary material}

Additional supplementary material may be found in the online version of this article at www.rcpjournals.org/clinmedicine: S1 - Pharmacological agents that can be used for functional gastrointestinal disorders.

\section{References}

1 Drossman DA. Functional gastrointestinal disorders: history, pathophysiology, clinical features and Rome IV. Gastroenterology 2016:150:1262-79.e2.

2 Sperber AD, Bangdiwala SI, Drossman DA et al. Worldwide prevalence and burden of functional gastrointestinal disorders, results of Rome Foundation global study. Gastroenterology 2020 [Epub ahead of print].

3 Jones MP, Crowell MD, Olden KW, Creed F. Functional gastrointestinal disorders: an update for the psychiatrist. Psychosomatics 2007:48:93-102.

4 Shivaji UN, Ford AC. Prevalence of functional gastrointestinal disorders among consecutive new patient referrals to a gastroenterology clinic. Frontline Gastroenterol 2014;5:266-71.

5 Aziz I, Palsson OS, Tornblom H et al. The prevalence and impact of overlapping rome iv-diagnosed functional gastrointestinal disorders on somatization, quality of life, and healthcare utilization: a cross-sectional general population study in three countries. Am J Gastroenterol 2018;113:86-96. 
6 Mahon J, Lifschitz C, Ludwig T et al. The costs of functional gastrointestinal disorders and related signs and symptoms in infants: a systematic literature review and cost calculation for England. BMJ Open 2017;7:e015594

7 Petersen MW, Schroder A, Jorgensen T et al. Irritable bowel, chronic widespread pain, chronic fatigue and related syndromes are prevalent and highly overlapping in the general population: DanFunD. Sci Rep 2020;10:3273.

8 Spiegel B, Harris L, Lucak S et al. Developing valid and reliable health utilities in irritable bowel syndrome: results from the IBS PROOF Cohort. Am J Gastroenterol 2009:104:1984-91.

9 Black CJ, Drossman DA, Talley NJ, Ruddy J, Ford AC. Functional gastrointestinal disorders: advances in understanding and management. Lancet 2020;396:1664-74.

10 Ford AC, Mahadeva S, Carbone MF, Lacy BE, Talley NJ. Functional dyspepsia. Lancet 2020;396:1689-1702.

11 Walters JR, Pattni SS. Managing bile acid diarrhoea. Therap Adv Gastroenterol 2010;3:349-57.

12 Basnayake C, Kamm MA, Stanley A et al. Standard gastroenterologist versus multidisciplinary treatment for functional gastrointestinal disorders (MANTRA): an open-label, single-centre, randomised controlled trial. Lancet Gastroenterol Hepatol 2020;5:890-9.

13 Cho KO, Jo YJ, Song BK, Oh JW, Kim YS. Colon transit time according to physical activity and characteristics in South Korean adults. World J Gastroenterol 2013;19:550-5.

14 Johannesson E, Simren M, Strid H, Bajor A, Sadik R. Physical activity improves symptoms in irritable bowel syndrome: a randomized controlled trial. Am J Gastroenterol 2011;106:915-22.

15 Cremonini F, Camilleri M, Zinsmeister AR et al. Sleep disturbances are linked to both upper and lower gastrointestinal symptoms in the general population. Neurogastroenterol Motil 2009;21:128-35.

16 Song GH, Leng PH, Gwee KA, Moochhala SM, Ho KY. Melatonin improves abdominal pain in irritable bowel syndrome patients who have sleep disturbances: a randomised, double blind, placebo controlled study. Gut 2005:54:1402-7.

17 Ohlsson B. The role of smoking and alcohol behaviour in management of functional gastrointestinal disorders. Best Pract Res Clin Gastroenterol 2017;31:545-52.

18 Memedovich KA, Dowsett LE, Spackman E, Noseworthy T, Clement F. The adverse health effects and harms related to marijuana use: an overview review. CMAJ Open 2018;6:E339-46.

19 Huestis MA, Solimini R, Pichini S et al. Cannabidiol adverse effects and toxicity. Curr Neuropharmacol 2019;17:974-89.

20 Nilholm C, Larsson E, Roth B, Gustafsson R, Ohlsson B. Irregular dietary habits with a high intake of cereals and sweets are associated with more severe gastrointestinal symptoms in IBS patients. Nutrients 2019;11:1279.

21 Staudacher HM, Ralph FSE, Irving PM, Whelan K, Lomer MCE. Nutrient intake, diet quality, and diet diversity in irritable bowel syndrome and the impact of the low FODMAP diet. J Acad Nutr Diet 2020;120:535-47.

22 Marsh A, Eslick EM, Eslick GD. Does a diet low in FODMAPs reduce symptoms associated with functional gastrointestinal disorders? A comprehensive systematic review and meta-analysis. Eur ] Nutr 2016:55:897-906.

23 Molina-Infante J, Serra J, Fernandez-Banares F, Mearin F. The lowFODMAP diet for irritable bowel syndrome: Lights and shadows. Gastroenterol Hepatol 2016;39:55-65.

24 Bohn L, Storsrud S, Liljebo T et al. Diet low in FODMAPs reduces symptoms of irritable bowel syndrome as well as traditional dietary advice: a randomized controlled trial. Gastroenterology 2015;149:1399-407.e2.

25 Eswaran SL, Chey WD, Han-Markey T, Ball S, Jackson K. A randomized controlled trial comparing the low FODMAP diet vs modified NICE guidelines in US adults with IBS-D. Am J Gastroenterol 2016:111:1824-32.
26 Biesiekierski JR, Peters SL, Newnham ED et al. No effects of gluten in patients with self-reported non-celiac gluten sensitivity after dietary reduction of fermentable, poorly absorbed, short-chain carbohydrates. Gastroenterology 2013;145:320-8.e1-3.

27 Ford AC, Talley NJ, Spiegel BM et al. Effect of fibre, antispasmodics, and peppermint oil in the treatment of irritable bowel syndrome: systematic review and meta-analysis. BMJ 2008;337:a2313.

28 Ford AC, Harris LA, Lacy BE, Quigley EMM, Moayyedi P. Systematic review with meta-analysis: the efficacy of prebiotics, probiotics, synbiotics and antibiotics in irritable bowel syndrome. Aliment Pharmacol Ther 2018:48:1044-60.

29 Wen Y, Li J, Long Q et al. The efficacy and safety of probiotics for patients with constipation-predominant irritable bowel syndrome: A systematic review and meta-analysis based on seventeen randomized controlled trials. Int J Surg 2020;79:111-9.

30 Ford AC, Moayyedi P, Chey WD et al. American College of Gastroenterology monograph on management of irritable bowel syndrome. Am J Gastroenterol 2018;113(Suppl 2):1-18.

31 Ford AC, Quigley EM, Lacy BE et al. Effect of antidepressants and psychological therapies, including hypnotherapy, in irritable bowel syndrome: systematic review and meta-analysis. Am J Gastroenterol 2014;109:1350-65;quiz 66.

32 Claudino AM, Pike KM, Hay P et al. The classification of feeding and eating disorders in the ICD-11: results of a field study comparing proposed ICD-11 guidelines with existing ICD-10 guidelines. BMC Med 2019;17:93.

33 Lindfors $P$, Unge $P$, Arvidsson $P$ et al. Effects of gut-directed hypnotherapy on IBS in different clinical settings-results from two randomized, controlled trials. Am J Gastroenterol 2012;107:276-85.

34 Ljotsson B, Falk L, Vesterlund AW et al. Internet-delivered exposure and mindfulness based therapy for irritable bowel syndrome-a randomized controlled trial. Behav Res Ther 2010;48:531-9.

35 Bonnert M, Olen O, Lalouni $\mathrm{M}$ et al. Internet-delivered exposurebased cognitive-behavioral therapy for adolescents with functional abdominal pain or functional dyspepsia: a feasibility study. Behav Ther 2019;50:177-88.

36 van der Veek PP, van Rood YR, Masclee AA. Clinical trial: short- and long-term benefit of relaxation training for irritable bowel syndrome. Aliment Pharmacol Ther 2007:26:943-52.

37 Lindfors $\mathrm{P}$, Unge $\mathrm{P}$, Nyhlin $\mathrm{H}$ et al. Long-term effects of hypnotherapy in patients with refractory irritable bowel syndrome. Scand J Gastroenterol 2012:47:414-20.

38 Lindfors P, Unge P, Arvidsson P et al. Effects of gut-directed hypnotherapy on IBS in different clinical settings-results from two randomized, controlled trials. Am J Gastroenterol 2012;107: 276-85.

39 Ljotsson B, Hedman E, Lindfors P et al. Long-term follow-up of internet-delivered exposure and mindfulness based treatment for irritable bowel syndrome. Behav Res Ther 2011;49:58-61.

40 Drossman DA, Toner BB, Whitehead WE et al. Cognitive-behavioral therapy versus education and desipramine versus placebo for moderate to severe functional bowel disorders. Gastroenterology 2003;125:19-31.

41. Simren M, Tornblom H, Palsson OS, Whitehead WE. Management of the multiple symptoms of irritable bowel syndrome. Lancet Gastroenterol Hepatol 2017;2:112-22.

42 Kurlander JE, Drossman DA. Diagnosis and treatment of narcotic bowel syndrome. Nat Rev Gastroenterol Hepatol 2014;11:410-8.

43 Lewis-Fernandez R, Lam P, Lucak S et al. An open-label pilot study of duloxetine in patients with irritable bowel syndrome and comorbid major depressive disorder. J Clin Psychopharmacol 2016:36:710-5.

44 Kaplan A, Franzen MD, Nickell PV, Ransom D, Lebovitz PJ. An openlabel trial of duloxetine in patients with irritable bowel syndrome and comorbid generalized anxiety disorder. Int J Psychiatry Clin Pract 2014;18:11-5 
45 Saito YA, Almazar AE, Tilkes KE et al. Randomised clinical trial: pregabalin vs placebo for irritable bowel syndrome. Aliment Pharmacol Ther 2019:49:389-97.

46 Lee KJ, Kim JH, Cho SW. Gabapentin reduces rectal mechanosensitivity and increases rectal compliance in patients with diarrhoeapredominant irritable bowel syndrome. Aliment Pharmacol Ther 2005;22:981-8.

47 Hanauer SB, DuPont HL, Cooper KM, Laudadio C. Randomized, double-blind, placebo-controlled clinical trial of loperamide plus simethicone versus loperamide alone and simethicone alone in the treatment of acute diarrhea with gas-related abdominal discomfort. Curr Med Res Opin 2007;23:1033-43.

48 Garsed K, Chernova J, Hastings $M$ et al. A randomised trial of ondansetron for the treatment of irritable bowel syndrome with diarrhoea. Gut 2014;63:1617-25.

49 Black C], Burr NE, Camilleri M et al. Efficacy of pharmacological therapies in patients with IBS with diarrhoea or mixed stool pattern: systematic review and network meta-analysis. Gut 2020;69: 74-82.

50 Li J, Zhu W, Liu W, Wu Y, Wu B. Rifaximin for irritable bowel syndrome: a meta-analysis of randomized placebo-controlled trials. Medicine (Baltimore) 2016;95:e2534

51 Brenner DM, Sayuk GS, Gutman CR et al. Efficacy and safety of eluxadoline in patients with irritable bowel syndrome with diarrhea who report inadequate symptom control with loperamide: RELIEF phase 4 study. Am J Gastroenterol 2019;114:1502-11.

52 Tack J, Quigley E, Camilleri M, Vandeplassche L, Kerstens R. Efficacy and safety of oral prucalopride in women with chronic constipation in whom laxatives have failed: an integrated analysis. United European Gastroenterol J 2013;1:48-59.
53 Tack J, van Outryve M, Beyens G, Kerstens R, Vandeplassche L. Prucalopride (Resolor) in the treatment of severe chronic constipation in patients dissatisfied with laxatives. Gut 2009;58:357-65.

54 Lacy BE, Schey R, Shiff S] et al. Linaclotide in chronic idiopathic constipation patients with moderate to severe abdominal bloating: a randomized, controlled trial. PLoS One 2015:10:e134349.

55 Schoenfeld P, Lacy BE, Chey WD et al. Low-dose linaclotide (72 mug) for chronic idiopathic constipation: a 12-week, randomized, double-blind, placebo-controlled trial. Am J Gastroenterol 2018;113:105-14.

56 Chang L, Chey WD, Drossman D et al. Effects of baseline abdominal pain and bloating on response to lubiprostone in patients with irritable bowel syndrome with constipation. Aliment Pharmacol Ther 2016;44:1114-22.

57 Pittayanon R, Yuan Y, Bollegala NP et al. Prokinetics for functional dyspepsia. Cochrane Database Syst Rev 2018;10:CD009431.

58 Ford AC, Luthra P, Tack ] et al. Efficacy of psychotropic drugs in functional dyspepsia: systematic review and meta-analysis. Gut 2017:66:411-20.

59 Cheong PK, Ford AC, Cheung CKY et al. Low-dose imipramine for refractory functional dyspepsia: a randomised, double-blind, placebo-controlled trial. Lancet Gastroenterol Hepatol 2018;3:837-44.

60 Tack J, Ly HG, Carbone F et al. Efficacy of mirtazapine in patients with functional dyspepsia and weight loss. Clin Gastroenterol Hepatol 2016;14:385-92.e4.

Address for correspondence: Dr Asma Fikree, The Royal Hospital, Whitechapel Road, London E1 1FR, UK.

Email: asma.fikree@nhs.net

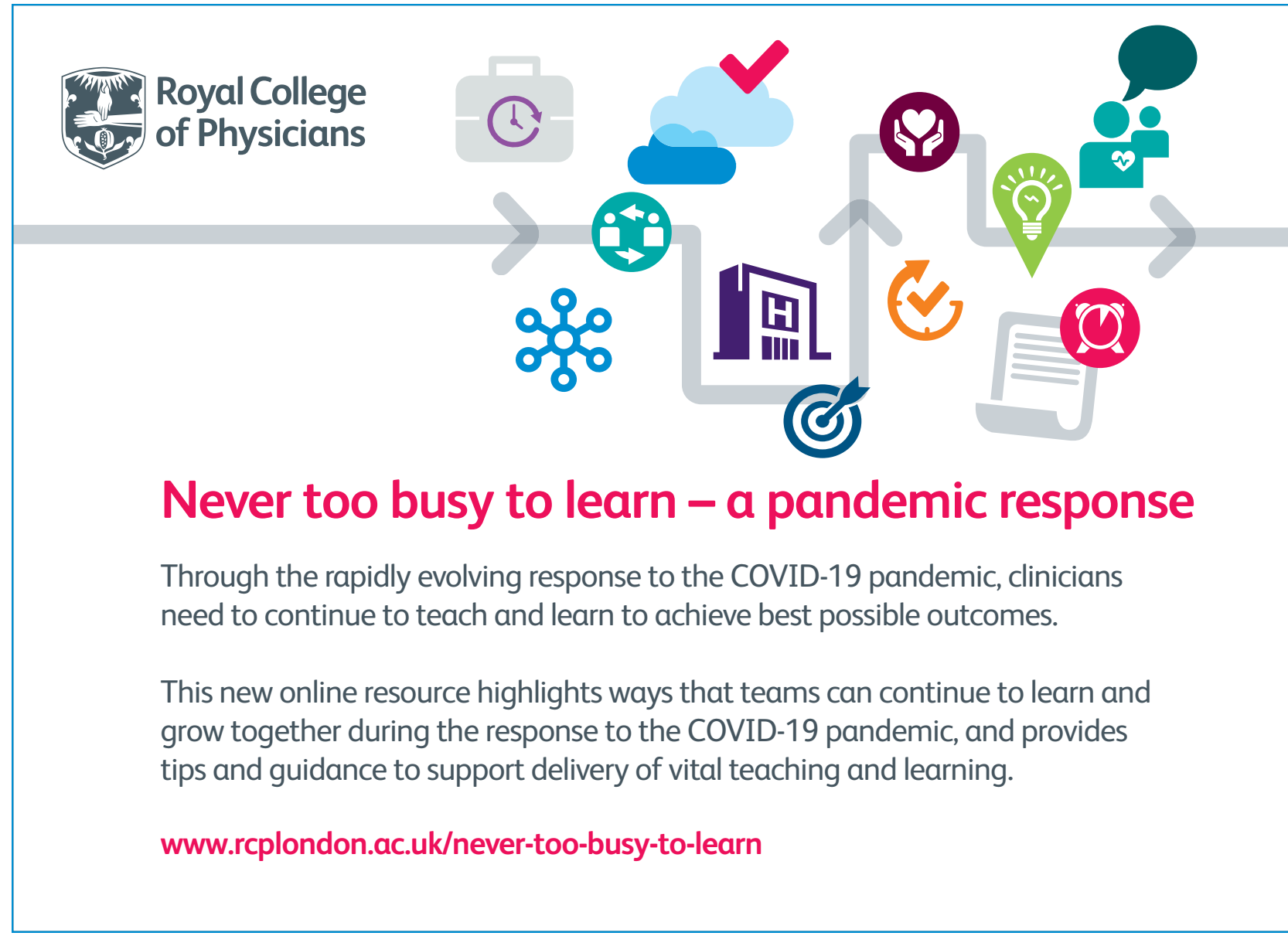

